\title{
A pilot study of near-infrared fluorescence guided surgery for primary tumor localization and lymph node mapping in colorectal cancer
}

\author{
Ying Cao ${ }^{1 \#}$, Peng Wang ${ }^{2,3 \#}$, Ziyang Wang ${ }^{1}$, Wei Zhang ${ }^{4}$, Qian Lu $^{1}$, Christopher J. Butch ${ }^{1 \wedge}$, \\ Nida El Islem Guissi ${ }^{1 \wedge}$, Qi You ${ }^{1}$, Huiming Cai ${ }^{1,5}$, Yongbin Ding ${ }^{2}$, Yiqing Wang ${ }^{1 \wedge}$ \\ ${ }^{1}$ Department of Biomedical Engineering, College of Engineering and Applied Sciences, State Key Laboratory of Analytical Chemistry for Life \\ Science, Nanjing University, Nanjing, China; ${ }^{2}$ Department of General Surgery, Pukou Branch of Jiangsu People's Hospital, Nanjing, China; \\ ${ }^{3}$ Department of general surgery, Jiangsu Province Geriatric Hospital, Nanjing, China; ${ }^{4}$ Jiangsu Testing and Inspection Institute for Medical Devices, \\ Nanjing, China; ${ }^{5}$ Nanjing Nuoyuan Medical Devices Co., Ltd, Nanjing, China \\ Contributions: (I) Conception and design: Y Wang; (II) Administrative support: H Cai; (III) Provision of study materials or patients: Y Ding, P Wang; \\ (IV) Collection and assembly of data: Y Cao, Z Wang; (V) Data analysis and interpretation: Y Wang, Y Cao, Z Wang; (VI) Manuscript writing: All \\ authors; (VII) Final approval of manuscript: All authors. \\ "These authors contributed equally to this work. \\ Correspondence to: Huiming Cai, Master; Yiqing Wang, PhD; Yongbin Ding, PhD. Department of Biomedical Engineering, College of Engineering \\ and Applied Sciences, Nanjing University, No. 22, Hankou Road, Gulou District, Nanjing 210093, China; Department of General Surgery, Pukou \\ Branch of Jiangsu People's Hospital, No. 166, Shanghe Street, Jiangpu Street, Pukou District, Nanjing 210000, China. Email: chm9166@aliyun.com; \\ njdyb@njmu.edu.cn; wangyiqing@nju.edu.cn.
}

Background: This pilot study aimed to evaluate the feasibility of near-infrared fluorescence imaging for primary tumor localization, lymph node mapping, and metastatic lymph node detection in colorectal cancer (CRC) using indocyanine green (ICG).

Methods: A total of 11 patients with CRC were prospectively enrolled. ICG $(25 \mathrm{mg}$ dissolved in $30 \mathrm{~mL}$ sterile water) was intravenously injected preoperatively, and the fluorescence intensity of the primary tumor, lymph nodes, and normal tissues, as well as the signal-to-background ratio (SBR) and contrast-to-noise ratio (CNR) were measured at $0.5,1,2,4$, and $24 \mathrm{~h}$ after ICG injection.

Results: The primary tumor could be located intraoperatively, and the tumor boundary was clear at 2-4 h. There was good contrast in the fluorescence intensity between tumor and normal tissues $(\mathrm{SBR}=2.11 \pm 0.36$, CNR $=8.74 \pm 0.35)$. The lymph node detection rate was $95 \%(38 / 40)$, and the SBR threshold of lymph nodes was 1.13 .

Conclusions: This pilot study showed that primary tumor localization and lymph node mapping in CRC is feasible using near-infrared fluorescence imaging technology, though metastatic lymph nodes cannot be discriminated from benign ones. In addition, cancer nodules missed by both white light mode and palpation by the surgeon were unexpectedly found, resulting in a change in the surgical prognosis in $9.1 \%(1 / 11)$ of patients.

Keywords: Near-infrared fluorescence imaging; indocyanine green (ICG); colorectal cancer (CRC); lymph node mapping; intravenous injection

Submitted Jul 07, 2021. Accepted for publication Aug 20, 2021.

doi: $10.21037 /$ atm-21-4021

View this article at: https://dx.doi.org/10.21037/atm-21-4021

^ ORCID: Christopher J. Butch, 0000-0003-3112-0470; Nida El Islem Guissi, 0000-0003-4425-7580; Yiqing Wang, 0000-0002-5626-8589. 


\section{Introduction}

Colorectal cancer (CRC) accounts for approximately $10 \%$ of cancer-related mortality in western countries (1) and is the leading cause of cancer-related deaths worldwide (2). The annual incidence of CRC is $38.7 / 100,000$ and the mortality rate is $13.9 / 100,000$ (3). As with many other types of solid tumors, surgery is the treatment of choice for CRC patients, extending the maximum patient survival time by 10 -fold (4). However, post-operative CRC patients exhibit a high local recurrence rate of $60 \%$ (5), in large part due to an incomplete resection rate of $30 \%$ (6). There are 2 main reasons for incomplete resection: positive tumor margin and incomplete mapping of proximal lymph nodes (7-9). Preoperatively, surgeons can employ imaging methods such as magnetic resonance imaging, ultrasound, and computed tomography (CT) to aid in diagnosis and localization, but during surgery, they mainly rely on visual and tactile perception to determine tumor boundaries and lymph node location. Lymph nodes are particularly difficult to locate in this manner, and discrimination between healthy and metastatic lymph nodes manually is impossible. Therefore, an intraoperative strategy allowing surgeons to visually determine positive tumor margins and detect metastatic lymph nodes intraoperatively and in real time is urgently needed to improve the prognosis of CRC patients.

Near-infrared fluorescence image-guided surgery (NIRFGS) is an emerging technology which allows surgeons to determine the tumor margins of many cancer types with greater accuracy and nanomolar detection sensitivity $(10,11)$. Compared with other optical wavelengths, near-infrared (NIR) fluorescence has deeper tissue penetration and lower background autofluorescence (12-14), allowing greater signal-to-background ratio (SBR). NIRFGS was first applied to the intraoperative localization of sentinel lymph nodes (15), greatly improving the sentinel lymph node detection rate up to $95 \%$ (16). The technique was then expanded to tumor localization, with excellent results in cancers such as liver $(17,18)$ and lung cancer $(19-21)$.

Indocyanine green (ICG) is currently the only NIR fluorescent tracer by approved by both the Chinese and US Food and Drug Administrations (CFDA and FDA) for use in humans $(22,23)$. In many cancer types, ICG has been reported to accumulate preferentially within solid tumors compared to surrounding normal tissue, thus allowing precise margin determination $(21,24,25)$. In a typical NIRFGS procedure, ICG is administered intravenously approximately 12 hours prior to the surgical procedure, and allows for a preoperative period prior to the surgical procedure. This preoperative period is intended to optimize the SBR due to the preferential accumulation of ICG in the tumor and its more rapid clearance from healthy tissue $(26,27)$. Local injection to the tumor site has also been explored, though it tends to exhibit increased background interference due to diffusion.

In CRC surgery, NIRFGS provides a well-established and reliable method to assess anastomotic perfusion, and its use can reduce the incidence of anastomotic leakage (28-31). However, for primary tumor localization of CRC, there are only a few studies, and all of them use the method of local injection of ICG (32-34). For lymph node mapping, lymphography injection methods (local injection) are typically used, and no special imaging protocols have been reported (35). The goal of the present study was to evaluate NIRFGS in CRC surgery using the intravenous (i.v.) method of ICG injection. The aims were: (I) localization of the primary tumor and determination of the tumor margin; (II) mapping lymph nodes; and (III) detection of metastatic lymph nodes using NIRFGS in CRC surgery. To our knowledge, this work use i.v. injected ICG in tumor location and lymph node mapping in CRC surgery for the first time, which is based on the Enhanced Permeability and Retention (EPR) effect. Compared with reported results, our method shows better fluorescence image contrast and margin identification of tumors, as well as greater detection of proximal lymph nodes. These results suggest that adoption of i.v. injection methods in clinical NIRFGS for CRC treatment may further improve the cure rate of CRC patients on the basis of mechanical surgical resection. Here, we used $25 \mathrm{mg}$ of ICG dissolved in $30 \mathrm{ml}$ of sterile water $(25 \mathrm{mg} /$ person), and ICG was administered intravenously into patients and then evaluated by SBR and contrast-to-noise ratio (CNR) intraoperatively.

In this paper, new indications are explored and breakthroughs are made in ICG-based image navigation. Unlike the traditional second-window ICG, the first window ICG is creatively proposed, which effectively avoids the problem of intestinal metabolite contamination in ICG and has guiding significance for clinical practice. We present the following article in accordance with the STROBE reporting checklist (available at https://dx.doi. org/10.21037/atm-21-4021). 
Table 1 Patient characteristics

\begin{tabular}{lcc}
\hline Patient characteristics $(n=11)$ & Number & Relative \% \\
\hline Gender & 1 & 9.0 \\
Female & 10 & 91.0 \\
Male & 6 & 55.0 \\
Localization & 5 & 45.0 \\
Colon & Mean \pm SD: $62.0 \pm 9.0$ & Median, range: $65,49-76$ \\
Rectal & &
\end{tabular}

$\mathrm{SD}$, standard deviation.

\section{Methods}

\section{Study design}

The exclusion criteria included: age $<18$ years, hyperthyroidism, documented coronary heart disease, significant renal failure, allergic history, pregnancy, lactation, and extensive metastases. 11 patients were included in this study. There were 6 cases of colon cancer and 5 cases of rectal cancer (Table 1). All procedures performed in this study involving human participants were in accordance with the Declaration of Helsinki (as revised in 2013). The study was approved by ethics board of Pukou Branch of Jiangsu People's Hospital (No.: 2019-LY-kt081) and informed consent was taken from all the patients.

During the surgery, the standard laparoscopic procedure was performed (36). After excision, the primary tumor was photographed and recorded using an NIR fluorescence navigation system for open surgery (FLI-10B). Next, the surgeon proceeded to perform a routine lymph node dissection, following the lymph nodes that were visualized using FLI-10B to search for metastatic lymph nodes and other micrometastases. All samples were sent for histopathological examination.

\section{Surgery and ICG injection}

ICG (clinical grade) was purchased from Dandong Yichuang Pharmaceutical Co., Ltd., China. The ICG injection dose was $25 \mathrm{mg} /$ person $(25 \mathrm{mg}$, dissolved in $30 \mathrm{~mL}$ sterile water for injection), and i.v. infusion of ICG was performed for $30 \mathrm{~min}$. In the first patient, $25 \mathrm{mg}$ was injected intravenously $24 \mathrm{~h}$ before surgery, and the tumor fluorescence was lower than that of the surrounding normal tissue during exploration, showing inverse contrast. Subsequently, we adjusted the protocol to shorten the tumor observation time. Therefore, the first patient was excluded from further analysis. Finally, 4 time points, $0.5,1,2$, and 4 h, were selected as groups for primary tumor localization and lymph node mapping.

\section{NIR imaging system}

The FLI-10B fluorescence navigation system (Figure 1) for open surgery was manufactured and provided by Nanjing Nuoyuan Medical Devices Co., Ltd. The sponsor was responsible for transporting the equipment to the operating room designated by the department undertaking the clinical study. The FLI-10B imaging system uses laser excitation at $785 \mathrm{~nm}$ to excite tissue containing ICG, and collects fluorescence images at wavelengths between 800 and $950 \mathrm{~nm}$. The systems display 3 channels simultaneously to the surgeon: visible light, fluorescent signal intensity, and a merger of the 2 . The in-built quantitative analytical functions allow the surgeons to precisely measure the fluorescence intensity, size, and area of the region of interest (ROI) intraoperatively.

\section{Statistical analysis}

\section{Calculation and value of the SBR}

To measure the fluorescence intensity and SBR of tumors, 5 points were selected at the tumor center and the 3, 6, 9, and 12 o'clock positions, and 5 points were selected as comprehensively as possible in the surrounding normal tissues (Figure 2A,2B). The SBR was calculated as follows:

$$
\text { SBR }=\frac{\sum_{i=1}^{5} \text { Tumor }_{i}}{\sum_{i=1}^{5} \text { Normal }_{i}}
$$

Where $\mathrm{Normal}_{i}$ is fluorescence intensity of point $i$ in the 


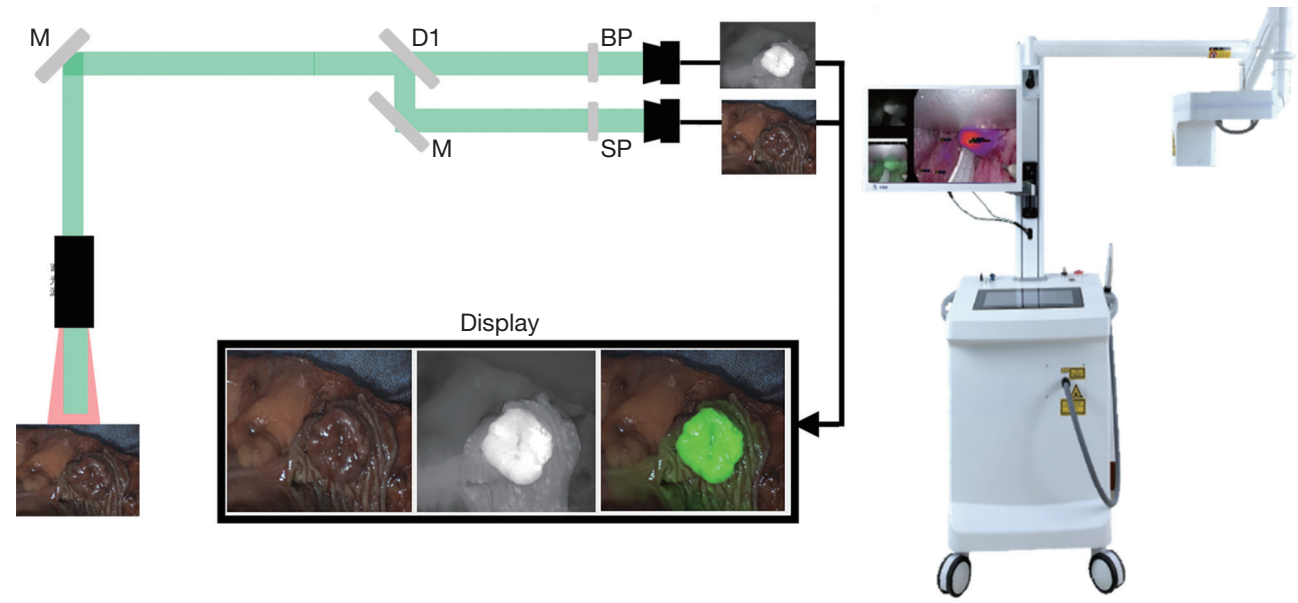

Figure 1 Schematic diagram of the imaging principle of the high-sensitivity, wide-field, and multimodal FLI-10B fluorescence imageguided surgical equipment and system.

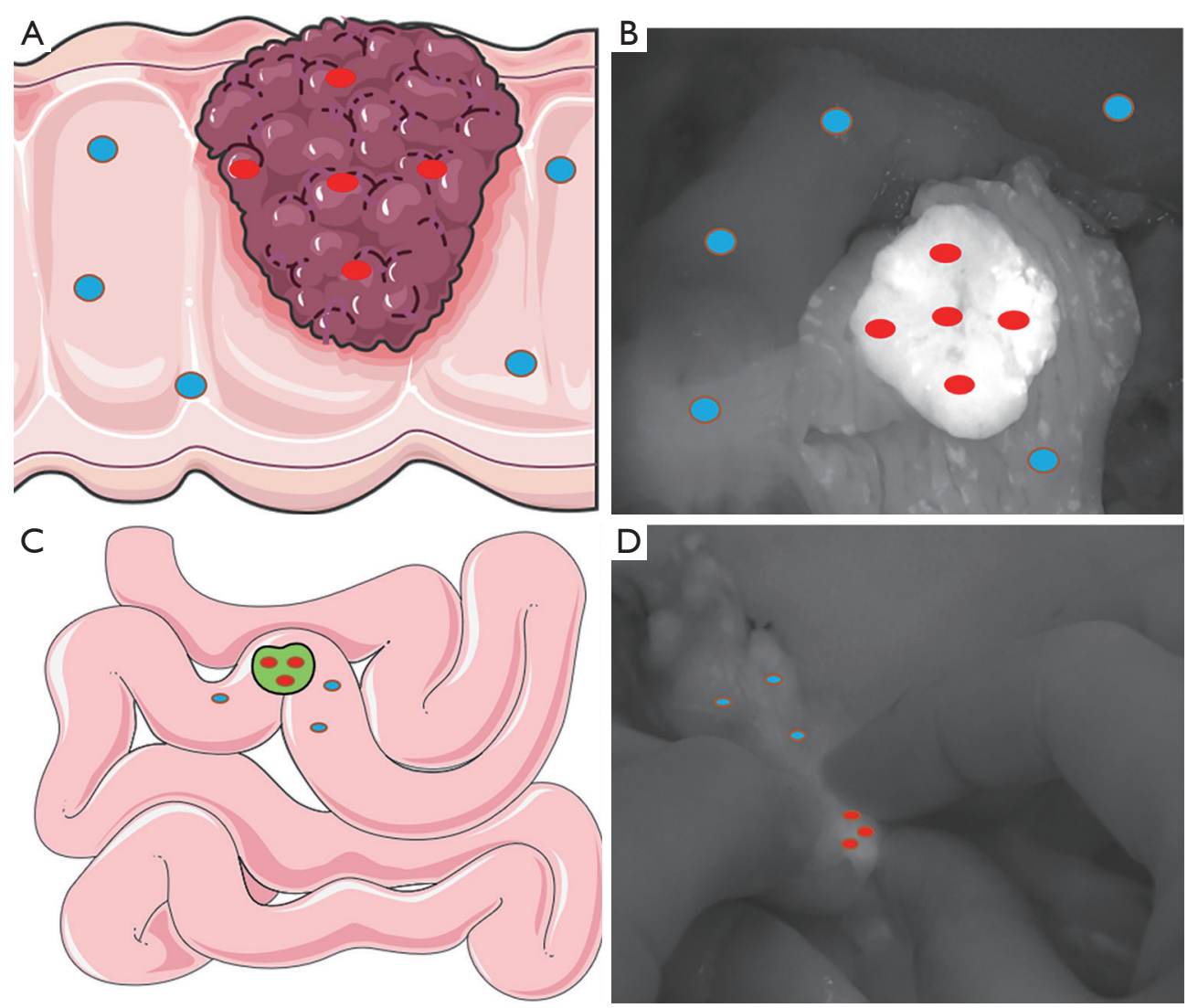

Figure 2 Near-infrared fluorescence navigator selected points. (A) Cartoon image, with 5 points on the tumor (red) and 5 points on normal tissue (blue). (B) Tumor image, with 5 points on the tumor (red) and 5 points on normal tissue (blue). (C) Cartoon image, where the green pattern is an intestinal lymph node, with the 3 brightest spots on the surface of the lymph node (red) and 3 points on normal tissue (blue). (D) Lymph node image, with the 3 brightest spots on the surface of the lymph node (red) and 3 points on normal tissue (blue). 
tumor, and $\mathrm{Normal}_{i}$ is fluorescence intensity of point $i$ in the normal tissue. All fluorescence intensity data points were selected and analyzed intrasurgically in the operating room on surgical navigation equipment.

\section{Calculation and value of the lymph node SBR}

To measure the fluorescence intensity and SBR of lymph nodes, we selected 3 points on each lymph node by highest fluorescence intensity and surrounding normal tissues (Figure 2C,2D). All fluorescence intensity data points were selected and analyzed intrasurgically in the operating room on surgical navigation equipment.

\section{Calculation of the intrasurgical detection limit}

The detection limit (also known as the minimum valid SBR) was calculated as:

$$
S B R_{\text {minimum }}=\frac{\text { Normal } \text { tissue }+3 * \sigma}{\text { Normal tissue }}
$$

Normal tissue is the average of the fluorescence intensity of normal tissue. $\sigma$ is the standard deviation of fluorescence intensity in the patient's normal tissue. All fluorescence intensity data points were selected and analyzed on site in the operating room on surgical navigation equipment.

\section{Contrast-to-noise ratio (CNR) calculation}

The CNR, an index reflecting the contrast and sharpness of the tumor boundary (37), was calculated by:

$$
C N R=\frac{\text { Tumor }- \text { Normal }}{\sigma_{\text {normal }}}
$$

Where $\sigma_{\text {normal }}$ is the standard deviation of normal tissue intensity. Furthermore, by the assumption that normal tissue intensities follow a normal distribution, the CNR correlates with the confidence level that the lesion's intensity is considered as higher than that of the normal tissues.

\section{Correlation calculations}

Correlation relationships between patients, surgical characteristics, and measured fluorescence data were assumed to be linear due to the small sample size. When multiple factors might have a simultaneous impact on measurements (e.g., incubation time and patient weight), we used partial correlation analysis and bivariate correlation analysis to construct a linear correlation between 2 variables based on an assumed linear regression of the third (e.g., planar regression in 3 dimensions).

\section{Results}

\section{Applicability of NIRFGS imaging-guided surgery in CRC}

In this study, the ICG injection dose was $25 \mathrm{mg} /$ person, and no allergic reactions were observed. The results are shown in Figure 3 and Table 2.

As shown in Figure 3A, 0.5 h after i.v. injection of ICG, the fluorescence intensity of the tumor was slightly higher than the surrounding tissues ( $\mathrm{SBR}=1.46 \pm 0.30, \mathrm{CNR}=4.73 \pm 1.61$, confidence level interval: 0.8554-0.9982), and the tumor boundary was not well defined.

At $1 \mathrm{~h}$ after injection (Figure $3 \mathrm{~B}$ ), the contrast was higher but it was still hard to differentiate the tumor boundary $(\mathrm{SBR}=1.89 \pm 0.43, \mathrm{CNR}=5.67 \pm 0.73$, confidence level interval: 0.9868-0.9984).

At $2 \mathrm{~h}$ after i.v. injection of ICG (Figure 3C), the tumor imaging effect was the best, and the tumor boundary was also the clearest $(\mathrm{SBR}=2.11 \pm 0.36, \mathrm{CNR}=8.74 \pm 0.35$, confidence level interval: 1.0000-1.0000).

At $4 \mathrm{~h}$ after i.v. injection of ICG (Figure 3D), the tumor imaging effect was relatively good, the tumor boundary was also relatively clear, but the tumor imaging effect had a large fluctuation $(\mathrm{SBR}=2.18 \pm 0.91, \mathrm{CNR}=4.20 \pm 2.31$, confidence level interval: 0.7850-0.9990).

At $24 \mathrm{~h}$ after i.v. injection of ICG (Figure $3 E$ ), the fluorescence intensity of the tumor was much lower than the surrounding tissues ( $\mathrm{SBR}=0.38 \pm 0.10$ ), but the tumor boundary remained clear.

Therefore, the imaging interval was adjusted to $2-4 \mathrm{~h}$ post-injection in subsequent experiments. The FLI-10B imaging system was used to acquire fluorescence intensity data and images. Images of the tumor were taken at each of the 5 imaging sites (Figure 3). Overall, the boundaries of the fluorescent tumors became clear after $1 \mathrm{~h}$, while the fluorescence boundaries were clearest at 2-4 h, showing the best recognition.

\section{Summary table of fluorescence intensity and SBR information of tumor and normal tissues}

Table 3 shows the statistics of all tumors by patient and time. To justify our uniform dose regimen of $25 \mathrm{mg} /$ person, we focused on analyzing body weight in relation to tumor and normal tissue fluorescence intensity and to the SBR. It was found that there was no significant correlation between body weight and SBR (correlation $=0.014$, significance $=0.976$, 2-tailed, partial correlations). At the same time, there was no correlation between body weight and the fluorescence 

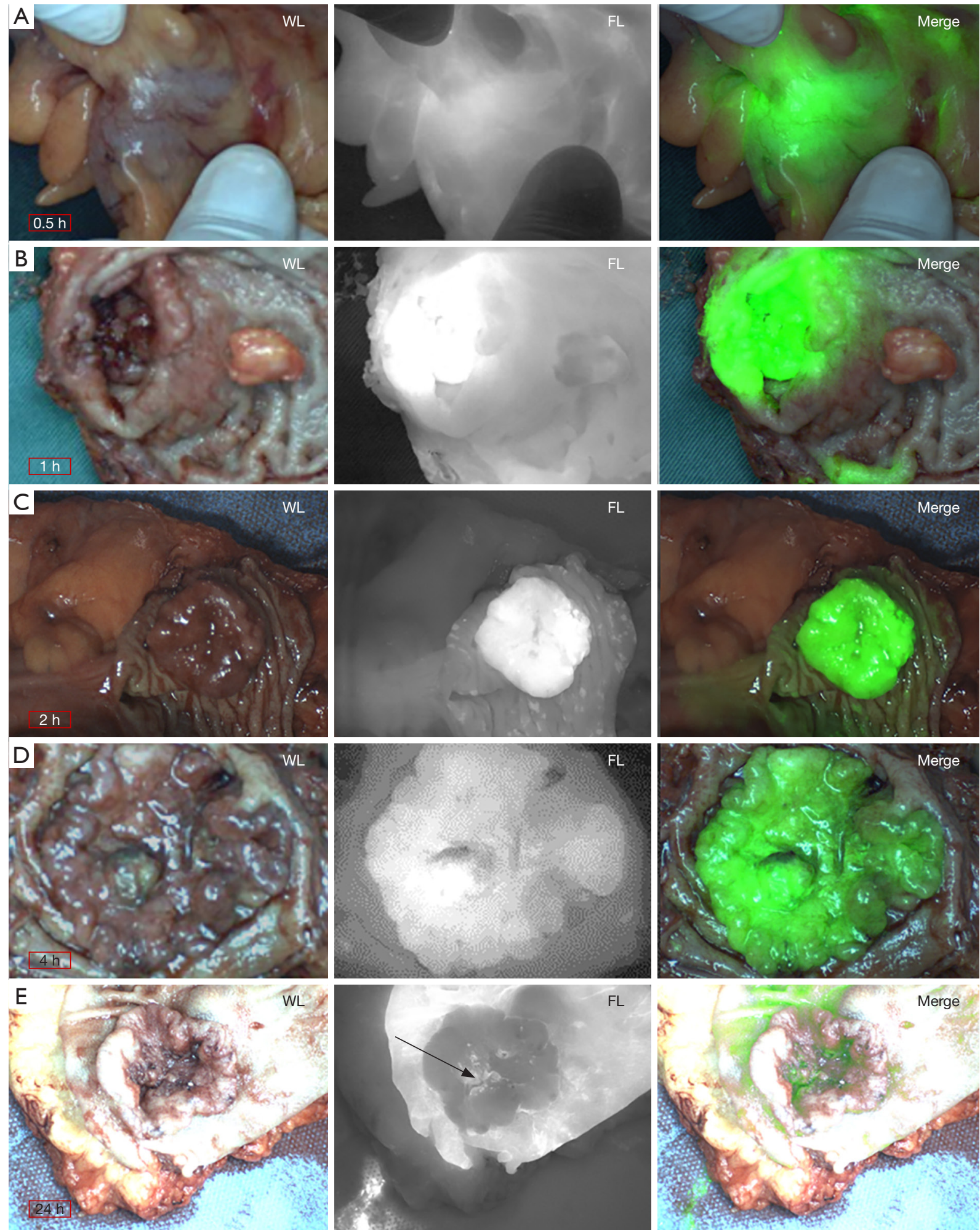

Figure 3 Typical tumor images of patients in different time imaging groups. (A) Imaging at $0.5 \mathrm{~h}$ after injection. (B) Imaging at $1 \mathrm{~h}$ after injection. (C) Imaging at $2 \mathrm{~h}$ after injection. (D) Imaging at $4 \mathrm{~h}$ after injection. (E) Imaging at $24 \mathrm{~h}$ after injection. WL, white light image; FL, fluorescence image (the arrow stands for tumors); merge, merged image. 
Table 2 SBR and CNR of tumors in the 4-time groups

\begin{tabular}{lccc}
\hline Time & SBR & CNR & Confidence level \\
\hline $0.5 \mathrm{~h}$ & $1.46 \pm 0.30$ & $4.73 \pm 1.61$ & $0.8554-0.9982$ \\
$1 \mathrm{~h}$ & $1.89 \pm 0.43$ & $5.67 \pm 0.73$ & $0.9868-0.9984$ \\
$2 \mathrm{~h}$ & $2.11 \pm 0.36$ & $8.74 \pm 1.07$ & $1.0000-1.0000$ \\
$4 \mathrm{~h}$ & $2.18 \pm 0.91$ & $4.20 \pm 2.31$ & $0.7850-0.9990$ \\
$24 \mathrm{~h}$ & $0.38 \pm 0.10$ & $-2.62 \pm 0.20$ & $0.0000-0.0000$ \\
\hline
\end{tabular}

SBRs and CNRs are shown as mean value \pm standard deviation. SBR, tumor signal-to-background ratio; CNR, contrast-to-noise ratio.

Table 3 Comparison of fluorescence intensity and SBR between tumor and normal tissues

\begin{tabular}{lccccccccc}
\hline Patient No. & Gender & Time $(\mathrm{h})$ & Weight, kg & Cancer type & Fl of tumor & Fl of normal tissue & SBR & CNR & Confidence level \\
\hline 1 & Female & 0.5 & 70 & $\mathrm{C}$ & $141.20 \pm 21.65$ & $106.20 \pm 7.19$ & $1.33 \pm 0.22$ & $4.87 \pm 3.01$ & $0.7517-1.0000$ \\
2 & Male & 0.5 & 73 & $\mathrm{C}$ & $178.80 \pm 14.72$ & $114.60 \pm 13.99$ & $1.56 \pm 0.23$ & $4.59 \pm 1.05$ & $0.9357-0.9949$ \\
3 & Male & 1 & 70 & $\mathrm{R}$ & $226.00 \pm 13.27$ & $148.40 \pm 14.74$ & $1.52 \pm 0.18$ & $5.26 \pm 0.90$ & $0.9706-0.9977$ \\
4 & Male & 1 & 75 & $\mathrm{C}$ & $164.73 \pm 12.18$ & $91.53 \pm 13.61$ & $1.80 \pm 0.30$ & $5.38 \pm 0.89$ & $0.9772-0.9981$ \\
5 & Male & 1 & 70 & $\mathrm{C}$ & $223.00 \pm 0.71$ & $99.20 \pm 19.49$ & $2.25 \pm 0.44$ & $6.35 \pm 0.04$ & $0.9968-0.9984$ \\
6 & Male & 2 & 90 & $\mathrm{C}$ & $122.73 \pm 11.01$ & $65.53 \pm 6.46$ & $1.87 \pm 0.25$ & $8.86 \pm 1.71$ & $1.0000-1.0000$ \\
7 & Male & 2 & 75 & $\mathrm{R}$ & $133.27 \pm 13.09$ & $58.27 \pm 8.99$ & $2.29 \pm 0.43$ & $8.34 \pm 1.56$ & $1.0000-1.0000$ \\
8 & Male & 2 & 78 & $\mathrm{C}$ & $174.80 \pm 20.17$ & $83.00 \pm 10.17$ & $2.11 \pm 0.35$ & $9.02 \pm 1.98$ & $1.0000-1.0000$ \\
9 & Male & 4 & 75 & $\mathrm{R}$ & $108.87 \pm 54.87$ & $58.07 \pm 12.30$ & $1.87 \pm 1.03$ & $4.13 \pm 4.46$ & $0.0000-1.0000$ \\
10 & Male & 5 & 62 & $\mathrm{R}$ & $43.53 \pm 6.76$ & $18.93 \pm 5.77$ & $2.30 \pm 0.79$ & $4.26 \pm 1.17$ & $0.9032-0.9932$ \\
11 & Male & 24 & 77 & $\mathrm{R}$ & $20.2 \pm 2.59$ & $53.4 \pm 12.66$ & $0.38 \pm 0.10$ & $-2.62 \pm 0.20$ & $0.0000-0.0000$ \\
\hline
\end{tabular}

Fls and SBRs are shown as mean value \pm standard deviation. SBR, tumor signal-to-background ratio; CNR, contrast-to-noise ratio; FI, fluorescence intensity.

intensity of tumor and normal tissues (correlation $=-0.269$, significance $=0.560$; correlation $=-0.377$, significance $=0.405$, 2-tailed, partial correlations). It can be seen from Table 3 that in the uniform dose regimen of $25 \mathrm{mg} /$ person, within the range of $5 \mathrm{~h}$, the fluorescence intensity of both tumor and normal tissues first increased and then decreased with time, while the SBR also had no significant unidirectional increasing or decreasing trend. Therefore, there were no significant correlations between the SBR, fluorescence intensity, and time (Pearson correlation $=-0.307$, significance $=0.460$, SBR with tumor intensity; Pearson correlation $=-0.677$, significance $=0.065$, SBR with normal tissue intensity; Pearson correlation $=0.497$, significance $=0.210$, 2-tailed, SBR with time, bivariate correlations).

\section{Optimal tumor visualization time for ICG NIRFGS for $C R C$}

To evaluate the feasibility of imaging the primary tumor of CRC, the SBR was calculated for each group (Figure $4 A$ ). The SBRs at $0.5,1,2,4$, and $24 \mathrm{~h}$ after ICG injection were $1.46 \pm 0.30,1.89 \pm 0.43,2.11 \pm 0.36,2.18 \pm 0.91$, and $0.38 \pm 0.10$, respectively (Table 2). The fluorescence signals of tumor and normal tissues were highest at $1 \mathrm{~h}$, and decreased gradually thereafter. In addition, we calculated that the CNR of each patient was greater than 3 , and the confidence level of each patient was greater than $96 \%$, demonstrating that the SBR of each tumor was statistically significant. From the mean value and image visibility of the SBR, the use of NIRFGS 

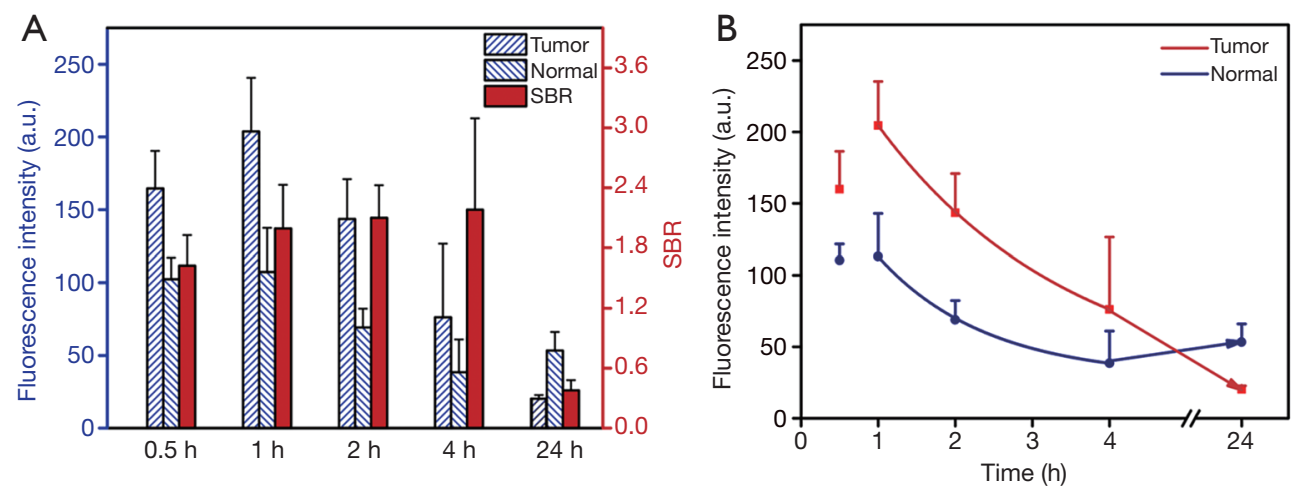

Figure 4 Fluorescence intensity. (A) Fluorescence intensity of tumor and normal tissues after indocyanine green (ICG) injection, and the signal-to-background ratio (SBR) between tumor and normal tissues. The SBR was highest at the $2 \mathrm{~h}$ measurement across all imaging times. (B) Fluorescence decay curves of ICG in tumor and normal tissues. The rate of tumor fluorescence metabolism was slower than that of normal tissue. $0.5 \mathrm{~h}(\mathrm{n}=10), 1 \mathrm{~h}(\mathrm{n}=15), 2 \mathrm{~h}(\mathrm{n}=15), 4 \mathrm{~h}(\mathrm{n}=10)$, and $24 \mathrm{~h}(\mathrm{n}=5)$.

for primary tumor localization in CRC was completely feasible. Taking into account the SBR $(2.11 \pm 0.36)$ and CNR $(8.74 \pm 1.07)$ of the tumor, and with the highest confidence level (1.0000) at this time, it was found that the tumor had the clearest margin and optimal visual imaging at $2 \mathrm{~h}$.

\section{Fluorescence decay of tumor and normal tissues}

After ICG injection, the fluorescence intensity of both tumor and normal tissues initially increased, with the highest observed level at $1 \mathrm{~h}$, and then decreased with time. ICG accumulation was preferential in the tumor, and the decay rate was slower than that of normal tissues (Figure $4 B$ ). Between 4 and $24 \mathrm{~h}$, the tumor fluorescence intensity still showed a downward trend, while the fluorescence intensity of normal tissues remained roughly constant or possibly even increased (perhaps due to excretion).

\section{Summary table of lymph node fuorescence intensity and SBR information}

Table 4 summarizes the gender, time, weight, and SBR for the 40 lymph node cases, 5 of which were positive lymph nodes, sorted in ascending order by the SBR for each node. The mean fluorescence intensity was $86.30 \pm 61.09$ for normal lymph nodes and $91.80 \pm 41.98$ for positive lymph nodes.

\section{Lymph node mapping}

Of the 11 CRC patients, 8 patients were sent for lymph node examination. A total of 40 lymph nodes were resected and histopathological analysis was performed. Among them, 5 lymph nodes were pathologically confirmed to be metastatic lymph nodes. All lymph nodes were detected from 0.5 to $5 \mathrm{~h}$ after ICG injection. A total of 38 of the 40 lymph nodes exhibited high fluorescence (Figure $5 A, 5 B$ ), while the remaining 2 lymph nodes had no fluorescence (Figure 5C,5D) and were detected by palpation by the surgeon. When the FLI-10B had a minimum valid SBR of 1.13 , the detection rate of lymph nodes was $95 \%$ (38/40). Although we could not achieve a detection rate of $100 \%$, the missed lymph nodes were all normal and had no adverse effects on lymph node dissection. The receiver operating characteristic (ROC) curve was plotted (Figure 5E), and the optimal detection threshold for metastatic lymph nodes was 2.50 according to the Youden index (38) when the sensitivity was $80.0 \%(4 / 5)$ and the specificity was $51.4 \%(18 / 35)$, and the positive predictive value (PPV) and negative predictive value (NPV) were $19.0 \%$ and $94.7 \%$, respectively (Figure $5 F$ ).

\section{Discussion}

\section{Metabolic characteristics of ICG in CRC}

In the present study, our primary objective was to assess the feasibility of NIRFGS for the visualization of the primary tumor in CRC. We used i.v. injection and obtained good imaging results $(\mathrm{SBR}=2.11 \pm 0.36, \mathrm{CNR}=8.74 \pm 0.35)$, with the clearest tumor borders and optimal localization at $2 \mathrm{~h}$ based on SBR, CNR, and the highest confidence level (1.0000). The feasibility of this method was successfully confirmed, 
Table 4 Summary table of lymph node statistics

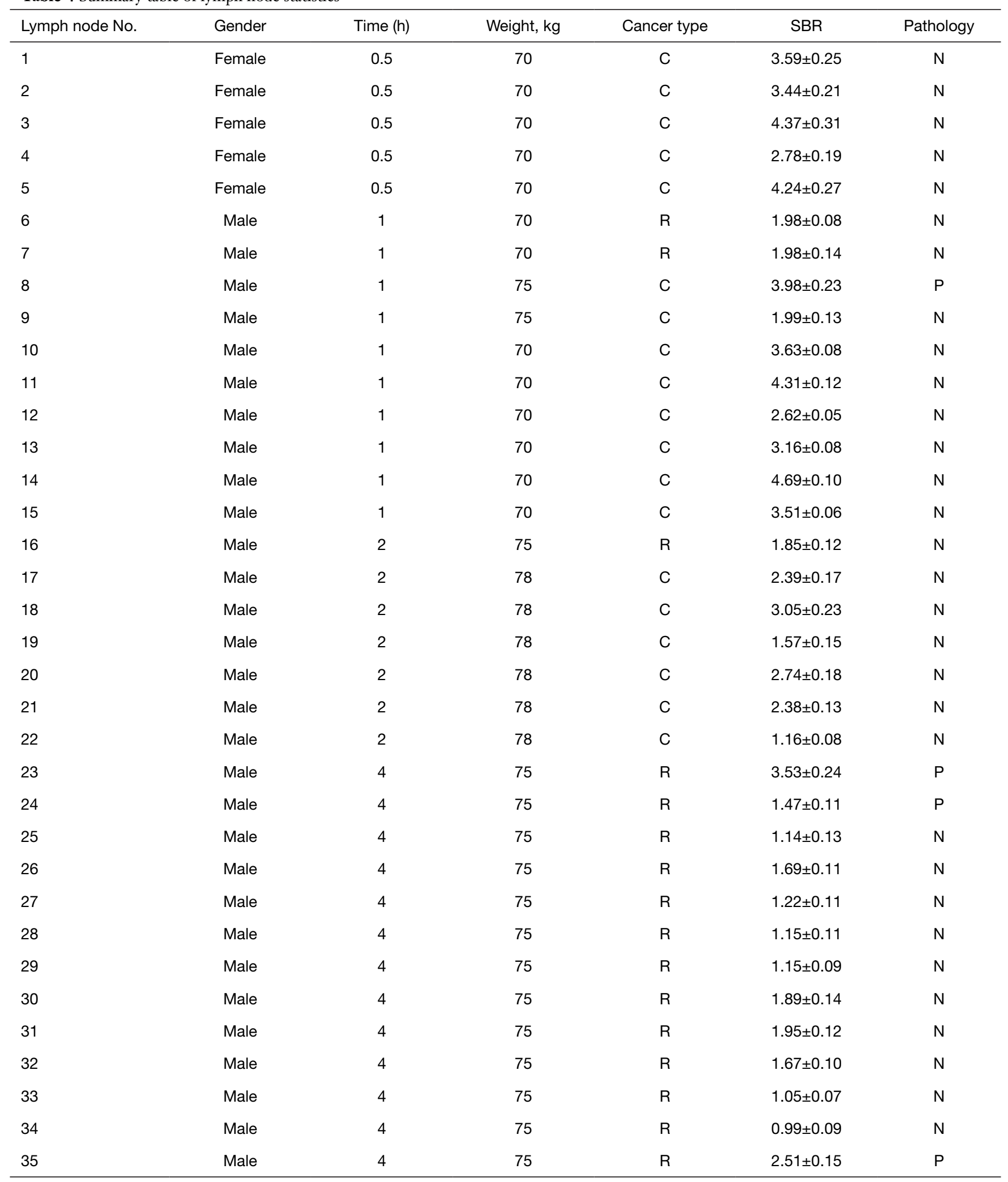

Table 4 (continued) 
Table 4 (continued)

\begin{tabular}{lccccc}
\hline Lymph node No. & Gender & Time $(\mathrm{h})$ & Weight, kg & Cancer type & SBR \\
\hline 36 & Male & 4 & 75 & $\mathrm{R}$ & $3.21 \pm 0.18$ \\
37 & Male & 5 & 62 & $\mathrm{R}$ & $6.84 \pm 1.34$ \\
38 & Male & 5 & 62 & $\mathrm{R}$ & $\mathrm{N}$ \\
39 & Male & 5 & 62 & $\mathrm{R}$ & $\mathrm{N}$ \\
40 & Male & 5 & 62 & $\mathrm{R}$ & $4.68 \pm 0.90$ \\
\hline
\end{tabular}

Fls and SBRs are shown as mean value \pm standard deviation. Fl, fluorescence intensity, SBR, lymph node signal-to-background ratio.
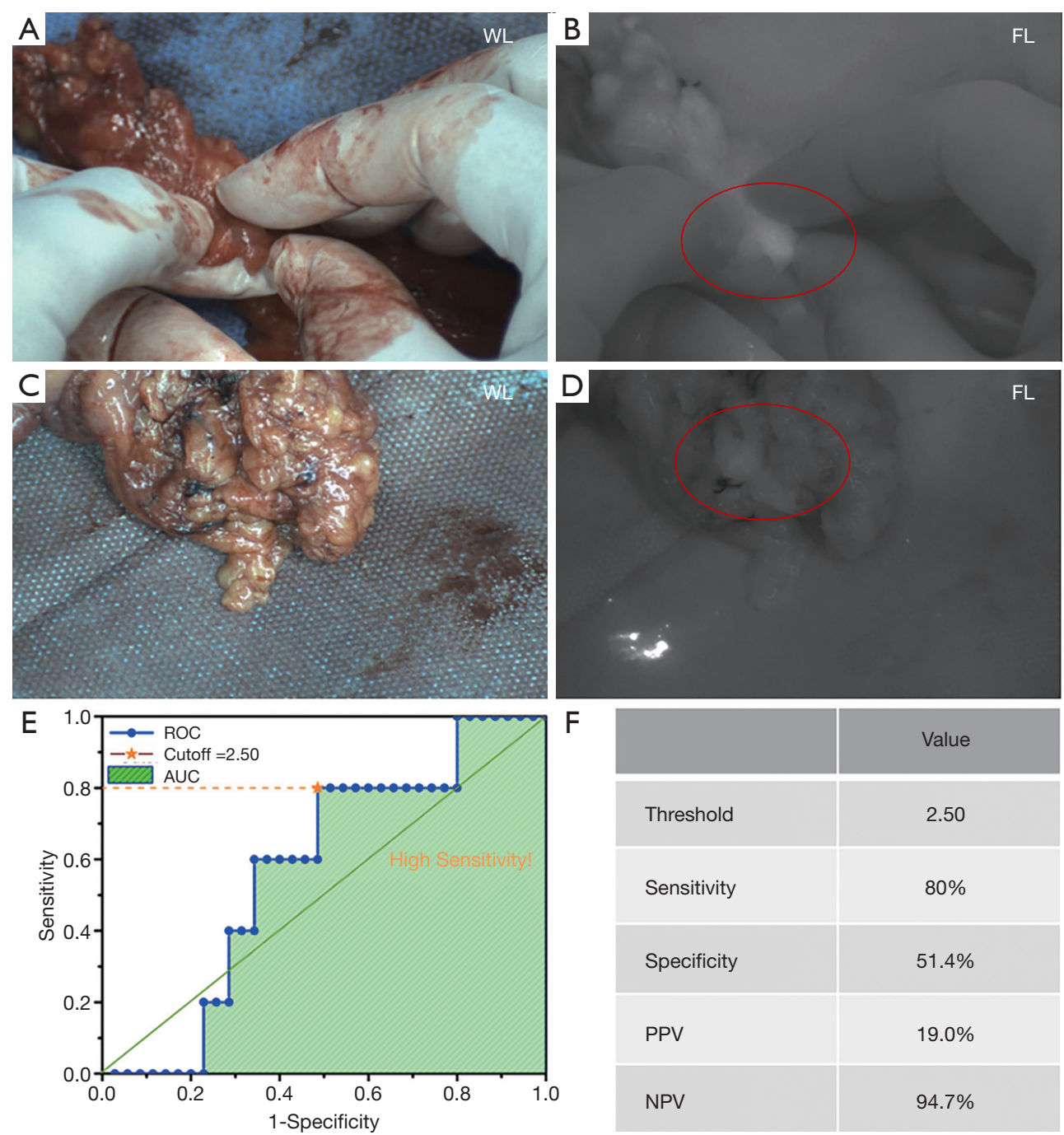

Figure 5 NIRFGS for lymph node mapping. (A,B) Typical detectable lymph node. (A) White light image. (B) Fluorescence image (red circle stands for detectable lymph node). (C,D) Typical missed lymph node. (C) White light image. (D) Fluorescence image (red circle stands for missed lymph node). (E) ROC curve. (F) Optimal predictive characteristics based on the Youden index. NIRFGS, near-infrared fluorescence image-guided surgery; ROC, receiver operating characteristic; WL, white light image; FL, fluorescence image. 
and the specific optimal time and dose regimen also require subsequent studies to expand sample size exploration. Therefore, for CRC tumors, we propose the concept of "the first window of ICG" here, and a time window of 2-4 h provides a stable observation period for CRC, while using the enhanced permeability and retention (EPR) effect of "fastin and fast-out" of CRC tumors and avoiding fluorescence contamination caused by intestinal metabolism. This can be seen from the fluorescence intensity decay curves of tumor and normal tissues (Figure 4B). Within $4 \mathrm{~h}$ after ICG injection, the fluorescence intensity of tumors was always higher than that of normal tissues, and the fluorescence intensity of tumors and normal tissues first increased and then decreased with time, both of which accumulated to the highest level at $1 \mathrm{~h}$, but the decay rate was different. The fluorescence intensity of the tumor decayed somewhat slower than that of normal tissue. During the $0.5-1 \mathrm{~h}$ increase in fluorescence intensity, the tumor fluorescence rise rate is $89.2 / \mathrm{h}$, while the fluorescence intensity rise rate of normal tissues was $5.2 / \mathrm{h}$, here, the rise rate represents the value of the increase in fluorescence intensity per unit time. The tumor fluorescence intensity rose faster than that of normal tissue, therefore, the perfusion rate of ICG in the tumor was faster.

Zeh et al. found that for ICG-based NIRFGS of solid tumors, there was no significant time dependence between 19 and $30 \mathrm{~h}$, thus providing a stable wide plateau for tumor visualization, called the "second window of ICG" (39). However, in our study, the tumor fluorescence was much weaker than that of the surrounding normal tissue at 24 $\mathrm{h}$ after injection (Figure $3 E$ ), which could be attributed to the fact that CRC has its own metabolic characteristics. At $0.5 \mathrm{~h}$ after injection, both the tumor and normal tissues were highly fluorescent. At 2-4 h after injection, the tumor exhibited the best SBR due to the preferential accumulation and slower decay rate relative to normal tissues. However, at $24 \mathrm{~h}$ after injection, the tumor was hypofluorescent relative to the background tissue, resulting in negative contrast. There are 3 factors which likely contribute to this phenomenon. First, due to the inherent characteristics of ICG metabolism and excretion, ICG is retained in the intestinal tract with excrement (40-43). Second, ICG can be reabsorbed via intestinal glands in the intestinal mucosa, resulting in continuous absorption and accumulation of ICG which has undergone biliary excretion in normal intestinal mucosal tissue. Third, due to differentiation, CRC tumor tissues disrupt the shape and function of intestinal mucosa resulting in decreased local reabsorption of ICG, so that the tumor fluorescence continues to decrease over time and is finally lower than that of normal tissues, with the result of reverse contrast. Therefore, avoiding hepatic metabolism needs to be considered when designing novel probes for CRC tumors, and probes should be metabolized by the kidney. Moreover, in the "first window of ICG", unlike the "second window of ICG", normal tissue is noisy and the boundary is not easy to define.

Based on our study and that of others, when using ICG for tumor surgical navigation, we need to consider the following three factors to image different tumors: (I) depending on the metabolic capacity of indocyanine green in tumors at different sites, indocyanine green was administered with different doses (17-21). (II) The ability of indocyanine green accumulation vary among different sites according to the vascular density of the tumor, resulting in different tumors correspond to different optimal imaging times (44). (III) Due to the inherent characteristics of digestive tract tumors, ICG can be reabsorbed through the glands in the digestive tract mucosa, resulting in continuous absorption and accumulation of ICG in normal mucosal tissues, and finally the phenomenon that the fluorescence of normal tissues is higher than that of background tissues (45). Moreover, a further development of the VITOM ${ }^{\circledR}$ exoscope with the feature of $3 \mathrm{D}$ visualization is now available but there are only a few reports with personal experiences $(46,47)$. Three-dimensional visualization is the development trend of endoscopic surgery, but the application combined with ICGNIFGS is still in the embryonic stage.

The CNR is a contrast evaluation parameter commonly used in MRI and CT images (37), and is calculated as (48):

$$
\text { CNR }=\frac{\text { Contrast }}{\text { Noise }}=\frac{\left|\mu_{1}-\mu_{2}\right|}{\sqrt{\sigma_{1}^{2}+\sigma_{2}^{2}}}
$$

In this study, we slightly modified the equation ssee Eq. [3]\}. In our calculation, $\mu_{1}$ and $\mu_{2}$ referred to the mean fluorescence intensity of the tumor and background normal tissues, respectively, while noise was defined as the standard deviation of background fluorescence intensity, namely $\sigma_{1} \cdot \sigma_{2}$. The standard deviation of tumor fluorescence intensity was not involved in our calculation to render a confidence interval on CNR. Under the premise that the background signals follow the normal distribution, the CNR here can correspond one-to-one with the confidence level with a difference between tumor and background, and there is a significant positive correlation between this value and the clarity of the tumor boundary, which can help the surgeon quantitatively determine the tumor boundary. 


\section{Advantages of i.v. injection of ICG}

Near-infrared fluorescence can effectively avoid autofluorescence of biological tissues, with the characteristics of large penetration depth, high signal-to-noise ratio and high safety, so it is expected to be used for intraoperative real-time navigation $(49,50)$. Indocyanine green is currently the only near-infrared fluorescent tracer approved by CFDA and FDA for use in humans. Methylene blue has an absorption peak of $665 \mathrm{~nm}$ and an emission peak of $688 \mathrm{~nm}$ (51). And indocyanine green absorption peak is $785 \mathrm{~nm}$, and the emission peak is $825 \mathrm{~nm}$. Compared with methylene blue, indocyanine green has a longer emission wavelength, deeper penetration depth and lower fluorescence background interference. Moreover, only a few clinical studies have reported the use of NIR fluorescence imaging to localize CRC primary tumors (26-28). However, these studies all used the method of local injection of ICG, which resulted in limitations such as unclear tumor boundary, poor image contrast, and background fluorescence interference. In addition, they did not perform data processing and quantitative analysis of tumor images. At the same time, although multi-wavelength near-infrared fluorescence imaging technology can achieve similar fluorescence imaging effect (52), its equipment is huge, expensive and immature. Furthermore, fluorescent molecules that can be applied to multi-wavelength fluorescence imaging technology are clinically unapproved molecules except ICG, such as fluorophores DEAC $(\lambda \mathrm{ex}=432 \mathrm{~nm}, \lambda \mathrm{em}=472 \mathrm{~nm})$, 5 -TAMRA $(\lambda e x=541 \mathrm{~nm}, \lambda \mathrm{em}=568 \mathrm{~nm})$ and CF633 $(\lambda e x$ $=630 \mathrm{~nm}, \lambda \mathrm{em}=650 \mathrm{~nm}$ ). Therefore, most fluorescence navigation devices on the market are designed for the NIR-I fluorescence of ICG (53).

In this study, the method of ICG i.v. injection was used to take advantage of the EPR effect of CRC tumors. As can be seen from Table 3, ICG preferentially accumulated in the tumor and was cleared from normal tissues faster, thus allowing for more precise tumor boundaries and better image contrast, resulting in a larger SBR and CNR.

\section{Lymph node mapping}

Sentinel lymph node imaging has achieved excellent imaging results in many tumors, with detection rates up to $95 \%$ (16), but sentinel lymph node biopsy is still in its infancy in CRC research $(54,55)$. Hirche et al. performed sentinel lymph node imaging in 26 CRC patients and performed intraoperative local injection of an average of $2.0 \mathrm{~mL}$
ICG solution $(5 \mathrm{mg} / \mathrm{mL})$. Among them, 15 were treated with conventional local injection, while the other 11 were treated with laparoscopic-assisted injection. On average, 1.7 lymph nodes were detected per patient, with a sensitivity of $82 \%$ (54). van der Pas et al. performed laparoscopic subserosal injection of $1-3 \mathrm{~mL}$ ICG solution $(2.5 \mathrm{mg} / \mathrm{mL})$ in 14 patients with colon cancer, and at least 1 lymph node was detected in each patient (55). All the above cases were local injections and had inconvenient injection operations. In our study, the detection rate of i.v. injection at $0.5-5 \mathrm{~h}$ was similar to that of a previous study (16), and the detection rate could reach 95\% (38/40) with an SBR threshold 1.13, using the noise-signal analysis method. Based on our study and others, we found that the main factors affecting the successful identification of sentinel lymph nodes by nearinfrared fluorescence imaging of colorectal cancer are: (I) the active targeting performance of the dye (56), (II) tissue penetration depth of dye fluorescence $(49,50)$. Although we could not achieve a detection rate of $100 \%$ in this paper, the 2 missed lymph nodes were normal lymph nodes, which did not affect the prognosis. Therefore, NIRFGS can be applied to both primary tumor localization and lymph node mapping simultaneously.

\section{Metastatic lymph node detection}

Because of the limitations of passive targeting of ICG, it is difficult to determine the metastatic status of lymph nodes, and the specificity of this dye was poor (45\%) (57). By plotting the ROC curve, we obtained an optimal threshold of 2.50 for lymph node diagnosis when the sensitivity and specificity were $80 \%(4 / 5)$ and $51.4 \%$ (18/35), respectively. That is, its accuracy and false negative rate is $80 \%$ and $48.6 \%$. In addition, from Table 4 the metastatic lymph node with the lowest fluorescence intensity had an SBR of 1.47 , which was still visible to the naked eye. Based on our results, we conclude that ICG can find all the lymph nodes as much as possible, but it cannot be used to distinguish metastatic lymph nodes.

\section{Other subclinical nodule exploration results}

In addition, with the assistance of FLI-10B, we found 3 cancerous nodules (Figure 6) which were not detected by neither preoperative CT scanning nor intraoperative palpation. This indicates the potential for clinical application of NIRFGS to explore or detect subclinical CRC metastases intraoperatively. 

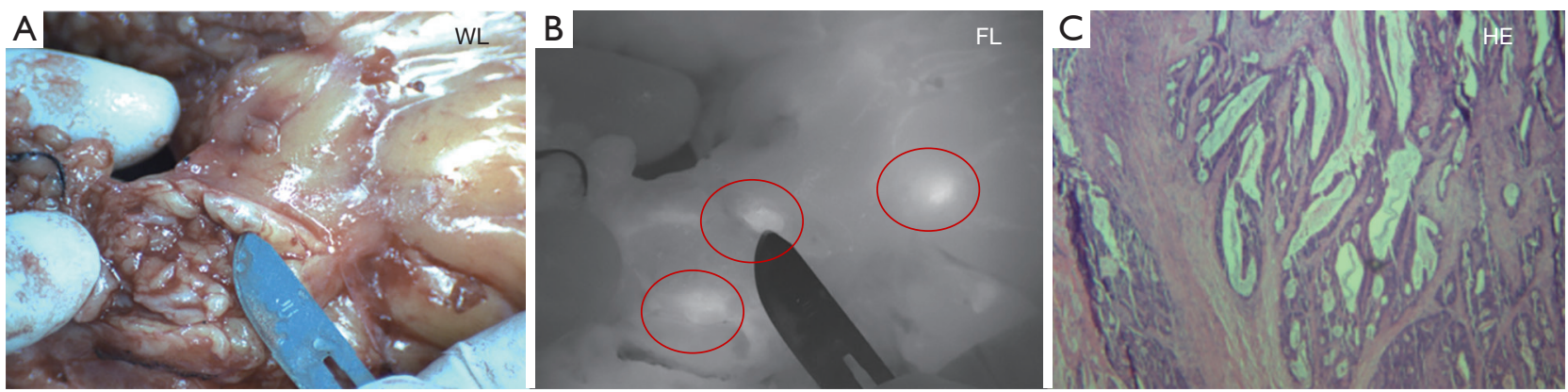

Figure 6 Incidental discovery of cancerous nodules and their multimodal imaging. (A) White light image. (B) Fluorescence image (red circles stand for 3 cancerous nodules). (C) Pathological images (the magnification is $200 \times$ ).

\section{Limitations of this study}

In future, to optimize this procedure, the patient sample size should be expanded, and more information such as the clinical stages of the patients and pathological stages of the tumors may be considered. There is a need to design active targeted fluorescent probes to replace ICG for the detection of positive lymph nodes.

\section{Conclusions}

A feasible surgical procedure was developed, and NIRFGS was demonstrated to be suitable not only for primary tumor localization, but also lymph node mapping in CRC. The "first window of ICG" (2-4 h post-injection) was found to be optimal for tumor imaging in CRC, and at the same window, the detection rate of lymph nodes reached $95 \%$. However, this method is not suitable for the diagnosis of positive lymph nodes. Moreover, one case showed the potential of ICG in detecting subclinical metastases, which changed the prognosis of the patient. Overall, these findings demonstrate the potential of NIRFGS for tumor imaging and lymph node mapping in CRC.

\section{Acknowledgments}

Funding: This study was supported by grants from the State Commission of Science \& Technology of China (Grant 2016YFC0104100), the National Natural Science Foundation of China (Grant 81901843), and the Jiangsu Province Science \& Technology Department (BE2016731 and BE2018698). YW acknowledges the "Jiangsu SpeciallyAppointed Professor" award.

\section{Footnote}

Reporting Checklist: The authors have completed the STROBE reporting checklist. Available at https://dx.doi. org/10.21037/atm-21-4021

Data Sharing Statement: Available at https://dx.doi. org/10.21037/atm-21-4021

Conflicts of Interest: All authors have completed the ICMJE uniform disclosure form (available at https://dx.doi. org/10.21037/atm-21-4021). HC is the owner and CEO of Nanjing Nuoyuan Medical Devices Co., Ltd. CJB is the technical consultant of Nanjing Nuoyuan Medical Devices Co., Ltd. The other authors have no conflicts of interest to declare.

Ethical Statement: The authors are accountable for all aspects of the work in ensuring that questions related to the accuracy or integrity of any part of the work are appropriately investigated and resolved. All procedures performed in this study involving human participants were in accordance with the Declaration of Helsinki (as revised in 2013). The study was approved by ethics board of Pukou Branch of Jiangsu People's Hospital (No.: 2019-LY-kt081) and informed consent was taken from all the patients.

Open Access Statement: This is an Open Access article distributed in accordance with the Creative Commons Attribution-NonCommercial-NoDerivs 4.0 International License (CC BY-NC-ND 4.0), which permits the noncommercial replication and distribution of the article with the strict proviso that no changes or edits are made and the 


\section{Page 14 of 16}

original work is properly cited (including links to both the formal publication through the relevant DOI and the license). See: https://creativecommons.org/licenses/by-nc-nd/4.0/.

\section{References}

1. Kuipers EJ, Grady WM, Lieberman D, et al. Colorectal cancer. Nat Rev Dis Primers 2015;1:15065.

2. Liberale G, Vankerckhove S, Caldon MG, et al. Fluorescence Imaging After Indocyanine Green Injection for Detection of Peritoneal Metastases in Patients Undergoing Cytoreductive Surgery for Peritoneal Carcinomatosis From Colorectal Cancer: A Pilot Study. Ann Surg 2016;264:1110-5.

3. Siegel RL, Miller KD, Goding Sauer A, et al. Colorectal cancer statistics, 2020. CA Cancer J Clin 2020;70:145-64.

4. Aliperti LA, Predina JD, Vachani A, et al. Local and systemic recurrence is the Achilles heel of cancer surgery. Ann Surg Oncol 2011;18:603-7.

5. van der Stok EP, Spaander MCW, Grünhagen DJ, et al. Surveillance after curative treatment for colorectal cancer. Nat Rev Clin Oncol 2017;14:297-315.

6. Elferink MA, de Jong KP, Klaase JM, et al. Metachronous metastases from colorectal cancer: a population-based study in North-East Netherlands. Int J Colorectal Dis 2015;30:205-12.

7. Qaderi SM, Galjart B, Verhoef C, et al. Disease recurrence after colorectal cancer surgery in the modern era: a population-based study. Int J Colorectal Dis 2021. [Epub ahead of print]. doi: 10.1007/s00384-021-03914-w.

8. Liberale G, Galdon MG, Moreau M, et al. Ex vivo detection of tumoral lymph nodes of colorectal origin with fluorescence imaging after intraoperative intravenous injection of indocyanine green. J Surg Oncol 2016;114:348-53.

9. Liberale G, Vankerckhove S, Galdon MG, et al. Fluorescence imaging after intraoperative intravenous injection of indocyanine green for detection of lymph node metastases in colorectal cancer. Eur J Surg Oncol 2015;41:1256-60.

10. Landau MJ, Gould DJ, Patel KM. Advances in fluorescentimage guided surgery. Ann Transl Med 2016;4:392.

11. Wang Z, Ni K, Zhang X, et al. Method for Real-Time Tissue Quantification of Indocyanine Green Revealing Optimal Conditions for Near Infrared Fluorescence Guided Surgery. Anal Chem 2018;90:7922-9.

12. Keating J, Tchou J, Okusanya O, et al. Identification of breast cancer margins using intraoperative near-infrared

\section{Cao et al. Primary tumor localization and lymph node mapping}

imaging. J Surg Oncol 2016;113:508-14.

13. Reinhart MB, Huntington CR, Blair LJ, et al. Indocyanine Green: Historical Context, Current Applications, and Future Considerations. Surg Innov 2016;23:166-75.

14. Martirosyan NL, Eschbacher JM, Kalani MY, et al. Prospective evaluation of the utility of intraoperative confocal laser endomicroscopy in patients with brain neoplasms using fluorescein sodium: experience with 74 cases. Neurosurg Focus 2016;40:E11.

15. Troyan SL, Kianzad V, Gibbs-Strauss SL, et al. The FLARE intraoperative near-infrared fluorescence imaging system: a first-in-human clinical trial in breast cancer sentinel lymph node mapping. Ann Surg Oncol 2009;16:2943-52.

16. Jewell EL, Huang JJ, Abu-Rustum NR, et al. Detection of sentinel lymph nodes in minimally invasive surgery using indocyanine green and near-infrared fluorescence imaging for uterine and cervical malignancies. Gynecol Oncol 2014;133:274-7.

17. van der Vorst JR, Schaafsma BE, Hutteman M, et al. Nearinfrared fluorescence-guided resection of colorectal liver metastases. Cancer 2013;119:3411-8.

18. Ishizawa T, Zuker NB, Kokudo N, et al. Positive and negative staining of hepatic segments by use of fluorescent imaging techniques during laparoscopic hepatectomy. Arch Surg 2012;147:393-4.

19. Keating J, Newton A, Venegas O, et al. Near-Infrared Intraoperative Molecular Imaging Can Locate Metastases to the Lung. Ann Thorac Surg 2017;103:390-8.

20. Okusanya OT, Holt D, Heitjan D, et al. Intraoperative near-infrared imaging can identify pulmonary nodules. Ann Thorac Surg 2014;98:1223-30.

21. Holt D, Okusanya O, Judy R, et al. Intraoperative nearinfrared imaging can distinguish cancer from normal tissue but not inflammation. PLoS One 2014;9:e103342.

22. Martirosyan NL, Cavalcanti DD, Eschbacher JM, et al. Use of in vivo near-infrared laser confocal endomicroscopy with indocyanine green to detect the boundary of infiltrative tumor. J Neurosurg 2011;115:1131-8.

23. DSouza AV, Lin H, Henderson ER, et al. Review of fluorescence guided surgery systems: identification of key performance capabilities beyond indocyanine green imaging. J Biomed Opt 2016;21:80901.

24. Madajewski B, Judy BF, Mouchli A, et al. Intraoperative near-infrared imaging of surgical wounds after tumor resections can detect residual disease. Clin Cancer Res 2012;18:5741-51.

25. Kosaka N, Mitsunaga M, Longmire MR, et al. Near 
infrared fluorescence-guided real-time endoscopic detection of peritoneal ovarian cancer nodules using intravenously injected indocyanine green. Int $\mathrm{J}$ Cancer 2011;129:1671-7.

26. DeLong JC, Hoffman RM, Bouvet M. Current status and future perspectives of fluorescence-guided surgery for cancer. Expert Rev Anticancer Ther 2016;16:71-81.

27. Jiang JX, Keating JJ, Jesus EM, et al. Optimization of the enhanced permeability and retention effect for nearinfrared imaging of solid tumors with indocyanine green. Am J Nucl Med Mol Imaging 2015;5:390-400.

28. Cahill RA, Ris F, Mortensen NJ. Near-infrared laparoscopy for real-time intra-operative arterial and lymphatic perfusion imaging. Colorectal Dis 2011;13 Suppl 7:12-7.

29. Keller DS, Ishizawa T, Cohen R, et al. Indocyanine green fluorescence imaging in colorectal surgery: overview, applications, and future directions. Lancet Gastroenterol Hepatol 2017;2:757-66.

30. Ris F, Liot E, Buchs NC, et al. Multicentre phase II trial of near-infrared imaging in elective colorectal surgery. Br J Surg 2018;105:1359-67.

31. Watanabe J, Ishibe A, Suwa Y, et al. Indocyanine green fluorescence imaging to reduce the risk of anastomotic leakage in laparoscopic low anterior resection for rectal cancer: a propensity score-matched cohort study. Surg Endosc 2020;34:202-8.

32. Nagata J, Fukunaga Y, Akiyoshi T, et al. Colonic Marking With Near-Infrared, Light-Emitting, Diode-Activated Indocyanine Green for Laparoscopic Colorectal Surgery. Dis Colon Rectum 2016;59:e14-8.

33. Watanabe $M$, Murakami $M$, Ozawa $Y$, et al. Intraoperative Identification of Colonic Tumor Sites Using a NearInfrared Fluorescence Endoscopic Imaging System and Indocyanine Green. Dig Surg 2017;34:495-501.

34. Zako T, Ito M, Hyodo H, et al. Extra-luminal detection of assumed colonic tumor site by near-infrared laparoscopy. Surg Endosc 2016;30:4153-9.

35. Emile SH, Elfeki H, Shalaby M, et al. Sensitivity and specificity of indocyanine green near-infrared fluorescence imaging in detection of metastatic lymph nodes in colorectal cancer: Systematic review and meta-analysis. J Surg Oncol 2017;116:730-40.

36. Lai JH, Law WL. Laparoscopic surgery for colorectal cancer. Br Med Bull 2012;104:61-89.

37. Baker ME, Dong F, Primak A, et al. Contrast-to-noise ratio and low-contrast object resolution on full- and lowdose MDCT: SAFIRE versus filtered back projection in a low-contrast object phantom and in the liver. AJR Am J Roentgenol 2012;199:8-18.

38. Youden WJ. Index for rating diagnostic tests. Cancer $1950 ; 3: 32-5$.

39. Zeh R, Sheikh S, Xia L, et al. The second window ICG technique demonstrates a broad plateau period for near infrared fluorescence tumor contrast in glioblastoma. PLoS One 2017;12:e0182034.

40. Shibasaki Y, Sakaguchi T, Hiraide T, et al. Expression of indocyanine green-related transporters in hepatocellular carcinoma. J Surg Res 2015;193:567-76.

41. Ishizawa T, Masuda K, Urano Y, et al. Mechanistic background and clinical applications of indocyanine green fluorescence imaging of hepatocellular carcinoma. Ann Surg Oncol 2014;21:440-8.

42. Onda N, Kimura M, Yoshida T, et al. Preferential tumor cellular uptake and retention of indocyanine green for in vivo tumor imaging. Int J Cancer 2016;139:673-82 .

43. Desmettre T, Devoisselle JM, Mordon S. Fluorescence properties and metabolic features of indocyanine green (ICG) as related to angiography. Surv Ophthalmol 2000;45:15-27.

44. Xie, Diya; Wang, Yuxin; Wang, Ziyang. Kinetics analysis of indocyanine green based on a novel mouse model to distinguish between tumor and inflammation. Analytical Methods (2019); 11:5704-5710.

45. Geibel JP. Secretion and absorption by colonic crypts. Annu Rev Physiol 2005;67:471-90.

46. Burkhardt BW, Csokonay A, Oertel JM. 3D-exoscopic visualization using the VITOM-3D in cranial and spinal neurosurgery. What are the limitations? Clin Neurol Neurosurg 2020;198:106101.

47. Oertel JM, Burkhardt BW. Vitom-3D for Exoscopic Neurosurgery: Initial Experience in Cranial and Spinal Procedures. World Neurosurg 2017;105:153-162.

48. Timischl F. The contrast-to-noise ratio for image quality evaluation in scanning electron microscopy. Scanning 2015;37:54-62.

49. Licha, K. Contrast agents for optical imaging. Contrast Agents II 2002;1-29.

50. Frangioni JV. In vivo near-infrared fluorescence imaging. Curr Opin Chem Biol 2003;7:626-34.

51. Matsui A, Tanaka E, Choi HS, et al. Real-time, nearinfrared, fluorescence-guided identification of the ureters using methylene blue. Surgery 2010;148: 78-86.

52. Yang L, Liu Z, Tan J. Multispectral imaging reveals hyper active TGF- $\beta$ signaling in colorectal cancer. Cancer Biol Ther 2018;19:105-112. 


\section{Page 16 of 16}

53. Joshi BP, Miller SJ, Lee CM. Multispectral endoscopic imaging of colorectal dysplasia in vivo. Gastroenterology 2012;143:1435-7.

54. Hirche C, Mohr Z, Kneif S, et al. Ultrastaging of colon cancer by sentinel node biopsy using fluorescence navigation with indocyanine green. Int $\mathrm{J}$ Colorectal Dis 2012;27:319-24.

55. van der Pas MH, Ankersmit M, Stockmann HB, et al. Laparoscopic sentinel lymph node identification in patients with colon carcinoma using a near-infrared dye: description of a new technique and feasibility study. J

Cite this article as: Cao Y, Wang P, Wang Z, Zhang W, Lu Q, Butch CJ, Guissi NEI, You Q, Cai H, Ding Y, Wang Y. A pilot study of near-infrared fluorescence guided surgery for primary tumor localization and lymph node mapping in colorectal cancer. Ann Transl Med 2021;9(16):1342. doi: 10.21037/atm21-4021

\section{Cao et al. Primary tumor localization and lymph node mapping}

Laparoendosc Adv Surg Tech A 2013;23:367-71.

56. Nishio N, van den Berg NS, van Keulen S. Optical molecular imaging can differentiate metastatic from benign lymph nodes in head and neck cancer. Nat Commun 2019;10:5044.

57. Lee JY, Thawani JP, Pierce J, et al. Intraoperative NearInfrared Optical Imaging Can Localize GadoliniumEnhancing Gliomas During Surgery. Neurosurgery 2016;79:856-71.

(English Language Editor: C. Betlzar) 